\title{
Robust and scalable scheme to generate large-scale entanglement webs
}

\section{$\operatorname{AUTHOR}(\mathrm{S}):$}

Fujii, Keisuke; Maeda, Haruki; Yamamoto, Katsuji

\section{CITATION:}

Fujii, Keisuke ...[et al]. Robust and scalable scheme to generate largescale entanglement webs. PHYSICAL REVIEW A 2011, 83(5): 050303.

ISSUE DATE:

2011-05-12

URL:

http://hdl.handle.net/2433/161770

RIGHT:

(C2011 American Physical Society 
PHYSICAL REVIEW A 83, 050303(R) (2011)

\title{
Robust and scalable scheme to generate large-scale entanglement webs
}

\author{
Keisuke Fujii, Haruki Maeda, and Katsuji Yamamoto \\ Department of Nuclear Engineering, Kyoto University, Kyoto 606-8501, Japan
}

(Received 23 February 2011; published 12 May 2011)

\begin{abstract}
We propose a robust and scalable scheme to generate an $N$-qubit $W$ state among separated quantum nodes (cavity-QED systems) by using linear optics and postselections. The present scheme inherits the robustness of the Barrett-Kok scheme [S. D. Barrett and P. Kok, Phys. Rev. A 71, 060310(R) (2005)]. The scalability is also ensured in the sense that an arbitrarily large $N$-qubit $W$ state can be generated with a quasipolynomial overhead $\sim 2^{\left.O\left[\log _{2} N\right)^{2}\right]}$. The process to breed the $W$ states, which we introduce to achieve the scalability, is quite simple and efficient and can be applied for other physical systems.
\end{abstract}

DOI: 10.1103/PhysRevA.83.050303

PACS number(s): 03.67.Bg, 03.65.Ud, 03.67.Hk, 42.50.Ex

Introduction. So far tremendous efforts have been paid for experimental realizations of quantum-information processing (QIP), and, for example, control of a few qubits has been performed in cavity QED, ion traps, etc. It, however, seems difficult to increase the number of qubits dramatically within a single physical system. In order to realize large-scale QIP, we have to develop a way to integrate individual physical systems scalably. Furthermore, for communication purposes, quantum information has to be shared among separated quantum nodes. To meet these requirements, distributed QIP, where stationary qubits are entangled by using flying qubits (photons), seems to be very promising [1-4]. A lot of protocols have been proposed so far for remote entangling operations and probabilistic two-qubit gates [5-7]. The Barrett-Kok scheme is particularly promising, since it is fully scalable and robust against experimental imperfections [6]. It is further studied for generating graph states efficiently [8]. Experiments of the remote entangling operations (or probabilistic two-qubit gates) between separated qubits have also been done in both atomic ensembles [9] and trapped single atoms [10]. They are important ingredients for fault-tolerant distributed quantum computation [6,11-13].

Multipartite entanglement is not only a key ingredient for quantum communication but also an important clue to understand the nature of quantum physics. There are a lot of classes of multipartite entanglement, for example, GHZ (Greenberger-Horne-Zeilinger) states [14], cluster states [15], and $W$ states [16]. Among them, the $W$ states,

$$
\left|W_{N}\right\rangle=\frac{1}{\sqrt{N}}(|100 \cdots 0\rangle+|010 \cdots 0\rangle \cdots+|000 \cdots 1\rangle,
$$

are quite robust in the sense that any pairs of qubits are still entangled, even if the rest of the qubits are discarded [17]. This weblike property is very fascinating as a universal resource, i.e., entanglement webs, for quantum communication. There are several protocols which use the $W$ states for quantum key distribution, teleportation, leader election, and information splitting [18]. Furthermore, inevitable decoherence in sharing the $W$ states can be counteracted by using a scheme of purification [19]. The preparation of the $W$ states by using optics has been discussed so far extensively both theoretically and experimentally [20]. It has been also discussed in other systems, such as cavity QED and ion traps [21]. Nevertheless none of them seems to be fully scalable. That is, the overhead required for sharing an $N$-qubit $W$ state scales exponentially in the number of qubits $N$ or the $W$ state is prepared in a single system, which cannot be used for quantum communication among separated quantum nodes.

In this paper, we develop a robust and scalable scheme to generate the $N$-qubit $W$ state by using separated cavity-QED systems and linear optics. The present scheme is scalable in the sense that an arbitrarily large $N$-qubit $W$ state can be generated among separated quantum nodes with only a quasipolynomial overhead $\left.\sim 2 O\left[\log _{2} N\right)^{2}\right]$. In the following, we first develop an efficient way to generate the four-qubit $W$ state $\left|W_{4}\right\rangle$ by following the concept of the Barrett-Kok scheme [6], which is quite robust against the experimental imperfections. The success probability to obtain the $\left|W_{4}\right\rangle$ is significantly high to be $1 / 2$. Then, by using the four-qubit $W$ states as seeds, we can breed an arbitrarily large $W$ state in an economical way, where the two $\left|W_{N}\right\rangle$ 's are converted to one $\left|W_{2(N-1)}\right\rangle$ probabilistically by accessing only two qubits. In contrast to classical webs, where a local connection does not result in a global web structure, this property of entanglement webs is a genuine quantum phenomenon. Even if the conversion fails, the two $\left|W_{N-1}\right\rangle$ 's are left and can be recycled. This breeding method is quite simple and economical and can be applied to other physical systems, such as polarization qubits in optics [20].

Four-qubit $W$ state (seeding). We consider four three-level atoms, each of which is embedded in a separated cavity. The two long-lived states of the atom, $|0\rangle$ and $|1\rangle$, are used as a qubit, where only the state $|1\rangle$ is coupled to the excited state $|e\rangle$, whose transition frequency is equal to that of the cavity mode (see Fig. 1). The output fields of the cavities are mixed with 50:50 beam splitters (BSs) and measured by photodetectors. The effective Hamiltonian of the system is given by

$$
H=\sum_{i=1}^{4} \frac{g_{i}}{2}\left(|1\rangle_{i i}\langle e| \hat{c}_{i}^{\dagger}+\text { H.c. }\right)-i \sum_{i=1}^{4} \kappa_{i} \hat{c}_{i}^{\dagger} \hat{c}_{i}
$$

where $g_{i}$ denotes the coupling between the $|1\rangle_{i} \leftrightarrow|e\rangle_{i}$ transition and the $i$ th cavity mode $\hat{c}_{i}$. The cavity photon leaks to the output mode with rate $2 \kappa_{i}\left(\kappa_{i}>g_{i}\right)$, which is treated as the non-Hermitian term by following the quantum jump approach [22]. For simplicity, the cavity parameters are set to $g_{i}=g$ and $\kappa_{i}=\kappa(i=1,2,3,4)$. As shown in Fig. 1, the output modes are mixed by using the four 50:50 BSs. Thus 


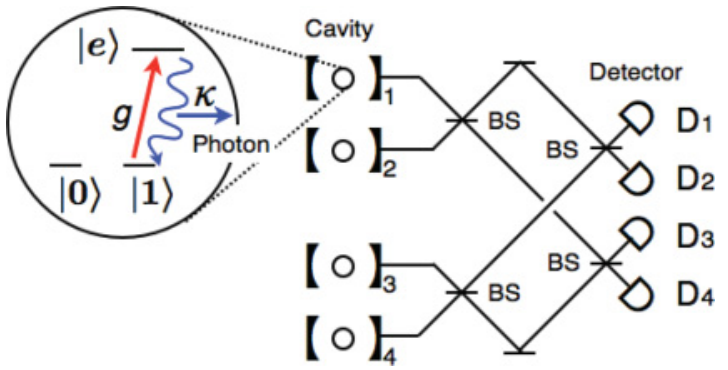

FIG. 1. (Color online) Three-level atoms are embedded in cavities. The two long-lived states $|0\rangle$ and $|1\rangle$ are used as a qubit, and the transition between the states $|1\rangle \leftrightarrow|e\rangle$ is coupled to the cavity mode. The output modes are mixed with four 50:50 BSs and measured by photodetectors $\mathrm{D}_{i}$.

the modes $\hat{a}_{i}$ of the four detectors $\mathrm{D}_{i}$ are given in terms of the cavity modes $\hat{c}_{i}$ by

$$
\begin{array}{ll}
\hat{a}_{1}=\left(\hat{c}_{1}+\hat{c}_{2}+\hat{c}_{3}+\hat{c}_{4}\right) / 2, & \hat{a}_{2}=\left(\hat{c}_{1}-\hat{c}_{2}+\hat{c}_{3}-\hat{c}_{4}\right) / 2, \\
\hat{a}_{3}=\left(\hat{c}_{1}+\hat{c}_{2}-\hat{c}_{3}-\hat{c}_{4}\right) / 2, & \hat{a}_{4}=\left(\hat{c}_{1}-\hat{c}_{2}-\hat{c}_{3}+\hat{c}_{4}\right) / 2 .
\end{array}
$$

The procedure to obtain the four-qubit $W$ state $\left|W_{4}\right\rangle$ is as follows. We first prepare the initial state of the atoms as $|\Psi(0)\rangle=(|0\rangle+|e\rangle)^{\otimes 4} / 4$ by using $\pi$ pulses. Then, we wait for a sufficiently long time $t_{w}$ to detect photons. Before proceeding to the second round, each qubit is flipped as $|0\rangle \leftrightarrow|1\rangle$, and the state $|1\rangle$ is excited to $|e\rangle$ by a $\pi$ pulse. Then we wait again for $t_{w}$ to detect photons. If three- and single-detector clicks, or vice versa, are observed at the first and second rounds, respectively, the $\left|W_{4}\right\rangle$ is obtained up to unimportant phase factors, which can be removed by using local operations.

Let us see in detail how the $\left|W_{4}\right\rangle$ is generated and calculate the success probability. For concreteness, we consider the case, where the $\mathrm{D}_{1}, \mathrm{D}_{2}$, and $\mathrm{D}_{3}$ are clicked at $t_{1}, t_{2}$, and $t_{3}\left(t_{1}<t_{2}<\right.$ $\left.t_{3}\right)$, respectively, in the first round. In the second round, the fourth detector is clicked at $t_{4}$. The state conditioned on the first three clicks is given up to normalization as

$$
\begin{aligned}
\left|\Psi\left(t_{1}, t_{2}, t_{3}\right)\right\rangle= & (2 \kappa)^{3 / 2} \hat{a}_{3} e^{-i H\left(t_{3}-t_{2}\right)} \hat{a}_{2} e^{-i H\left(t_{2}-t_{1}\right)} \hat{a}_{1} e^{-i H t_{1}}|\Psi(0)\rangle \\
= & \frac{(2 \kappa)^{3 / 2}}{8} \alpha\left(t_{3}\right) \alpha\left(t_{2}\right) \alpha\left(t_{1}\right)[\mathcal{W}(|1,0\rangle,|0,0\rangle) \\
& \left.+\alpha\left(t_{3}\right) \mathcal{W}(|1,0\rangle,|1,1\rangle)+\beta\left(t_{3}\right) \mathcal{W}(|1,0\rangle,|e, 0\rangle)\right],
\end{aligned}
$$

where $|a, b\rangle \quad(a \in\{0,1, e\}$ and $b \in\{0,1\})$ indicates the states of the atom $|a\rangle$ and photon $|b\rangle$, respectively, for the combination $\mathcal{W}(|A\rangle,|B\rangle) \equiv(|A\rangle|A\rangle|A\rangle|B\rangle-$ $|A\rangle|A\rangle|B\rangle|A\rangle-|A\rangle|B\rangle|A\rangle|A\rangle+|B\rangle|A\rangle|A\rangle|A\rangle) / 2$. The coefficients $\alpha(t)$ and $\beta(t)$ are the solutions to the Schrödinger equation:

$$
\begin{gathered}
\alpha(t)=-i g /\left(2 \sqrt{\kappa^{2}-g^{2}}\right)\left(-e^{\omega_{+} t}+e^{\omega_{-} t}\right), \\
\beta(t)=g^{2} /\left(4 \sqrt{\kappa^{2}-g^{2}}\right)\left(-e^{\omega_{+} t} / \omega_{+}+e^{\omega_{-} t} / \omega_{-}\right),
\end{gathered}
$$

where $\omega_{ \pm}=\left(-\kappa \pm \sqrt{\kappa^{2}-g^{2}}\right) / 2$. The probability of such an event is given by

$$
p\left(t_{1}, t_{2}, t_{3}\right)=\left|\left\langle\Psi\left(t_{1}, t_{2}, t_{3}\right) \mid \Psi\left(t_{1}, t_{2}, t_{3}\right)\right\rangle\right|^{2} .
$$

For the sufficiently long $t_{w}\left(\gg 1 /\left|\omega_{ \pm}\right|\right)$, the states $|e, 0\rangle$ and $|1,1\rangle$ decay to $|1,0\rangle$ incoherently. The postmeasurement state at $t_{w}$ is given by

$$
\rho\left(t_{w}\right)=\mathcal{N}\left[\rho_{\mathcal{W}}(|1,0\rangle,|0,0\rangle)+\left|\alpha\left(t_{\mathrm{w}}\right)\right|^{2}|1,0\rangle\left\langle 1,\left.0\right|^{\otimes 4}\right],\right.
$$

where $\rho_{\mathcal{W}}(|A\rangle,|B\rangle)=\mathcal{W}(|A\rangle,|B\rangle) \mathcal{W}(|A\rangle,|B\rangle)^{\dagger}$ and $\mathcal{N}=$ $(2 \kappa)^{3}\left|\alpha\left(t_{3}\right) \alpha\left(t_{2}\right) \alpha\left(t_{1}\right)\right|^{2} /\left[64 p\left(t_{1}, t_{2}, t_{3}\right)\right]$.

Before proceeding to the second round, each qubit is flipped as $|0\rangle \leftrightarrow|1\rangle$, and the state $|1\rangle$ is excited to $|e\rangle$ similarly to the first round. Then, the initial state of the second round is given by

$$
\rho^{\prime}(0)=\mathcal{N}\left[\rho_{\mathcal{W}}(|0,0\rangle,|e, 0\rangle)+\left|\alpha\left(t_{\mathrm{w}}\right)\right|^{2}|0,0\rangle\left\langle 0,\left.0\right|^{\otimes 4}\right] .\right.
$$

Since the first term has exactly one excitation, by observing the single detector click at $t_{4}$, the second term is removed in this round. Finally we obtain the four-qubit $W$ state $\left|W_{4}\right\rangle$ for the atoms. The joint probability for the first three clicks and the second single click is calculated as

$$
\begin{aligned}
p\left(t_{1}, t_{2}, t_{3}, t_{4}\right) & =p\left(t_{4} \mid t_{1}, t_{2}, t_{3}\right) p\left(t_{1}, t_{2}, t_{3}\right) \\
& =\operatorname{Tr}\left[2 \kappa \hat{a}_{4}^{\dagger} \hat{a}_{4} e^{-i H t_{4}} \rho^{\prime}(0) e^{i H t_{4}}\right] p\left(t_{1}, t_{2}, t_{3}\right) \\
& =(2 \kappa)^{4}\left|\alpha\left(t_{1}\right) \alpha\left(t_{2}\right) \alpha\left(t_{3}\right) \alpha\left(t_{4}\right)\right|^{2} / 256 .
\end{aligned}
$$

For sufficiently long $t_{w}\left(\gg 1 /\left|\omega_{ \pm}\right|\right)$, the success probability is calculated as

$$
\prod_{i=1}^{4} \int_{0}^{t_{\mathrm{W}}} d t_{i} \frac{(2 \kappa)^{4}}{256}\left|\alpha\left(t_{1}\right) \alpha\left(t_{2}\right) \alpha\left(t_{3}\right) \alpha\left(t_{4}\right)\right|^{2}=\frac{1}{256},
$$

where the sum over the orderings of $t_{1}, t_{2}$, and $t_{3}$ is also taken. By considering the cases for three detector clicks, $\left(D_{1}, D_{1}, D_{1}\right)$, $\left(\mathrm{D}_{1}, \mathrm{D}_{1}, \mathrm{D}_{2}\right)$, and so on, we obtain the total success probability $p=1 / 2$, which is unexpectedly high. This success probability can also be understood by the fact that the initial state $(|0\rangle+$ $|1\rangle)^{\otimes 4} / 4$ contains two types of the $W$ states (i.e., $|0001\rangle \ldots$ and $|1110\rangle \cdots)$ with each probability $1 / 4$. Then in the present setup, we can fully extract the $W$ states by virtue of the highly symmetric detector modes. This method inherits the robustness of the Barrett-Kok scheme [6]; the detector inefficiency and photon loss do not deteriorate the fidelity, but only decrease the success probability. The success probability scales like $p=$ $\left(\eta_{d} \eta_{l}\right)^{4} / 2$, where $\eta_{d}$ and $1-\eta_{l}$ denote the detector efficiency and photon loss rate, respectively. Other imperfections such as decoherence of the qubits, detector dark counts, and mode mismatchings would not deteriorate the fidelity crucially for a specific physical system such as the nitrogen-vacancy (NV)diamond system, as discussed in Ref. [6].

The above process to prepare the $\left|W_{4}\right\rangle$ is viewed as a single concatenation of entangling operation, $|1\rangle \rightarrow|10\rangle+|01\rangle$ and $|0\rangle \rightarrow|00\rangle$. It can be extended straightforwardly to generate an $N$-qubit $\left(N=2^{L}\right.$ with an integer $\left.L\right) W$ state $\left|W_{N}\right\rangle$ with probability $N / 2^{N-1}$ by using a similar setup. The detector modes are given by $\hat{a}_{j}^{(L)}=A_{i j}^{(L)} \hat{c}_{i} / \sqrt{N}$ in terms of an $N \times N$ matrix $A^{(L)}$ generated recursively by

$$
A^{(L+1)}=\left(\begin{array}{cc}
A^{(L)} & A^{(L)} \\
A^{(L)} & -A^{(L)}
\end{array}\right),
$$

where $A^{(0)}=1$. Then single and $N-1$ clicks, or vice versa, at the first and second rounds, respectively, result in the $\left|W_{N}\right\rangle$. 


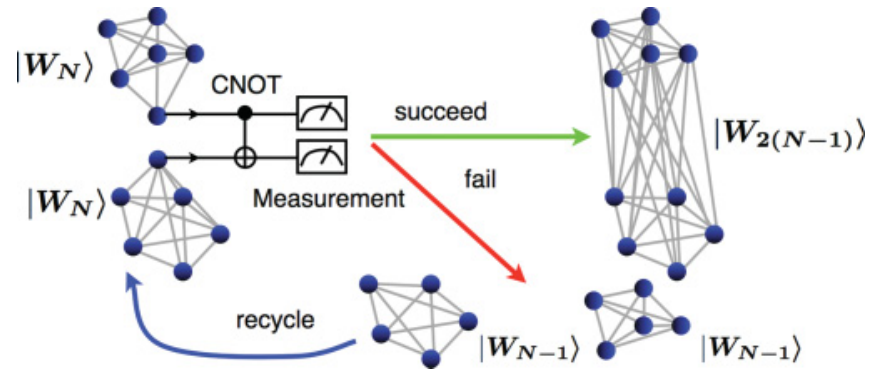

FIG. 2. (Color online) Economical breeding. Two $\left|W_{N}\right\rangle$ 's, which are depicted symbolically as circles connected with lines, are converted to a $\left|W_{2(N-1)}\right\rangle$ probabilistically. Even if the conversion fails, the two $\left|W_{N-1}\right\rangle$ 's are left and can be recycled.

More generally, if we observe $m$ and $N-m$ clicks at the first and second rounds, respectively, we can obtain the Dickesymmetric state [23]:

$$
\left.\left|D_{m, N-m}\right\rangle=\sum_{i} \mathcal{S}_{i}\left(|0\rangle^{\otimes m}|1\rangle^{\otimes N-m}\right\rangle\right) / \sqrt{\mathrm{C}_{N-m}^{m}},
$$

where $\left\{\mathcal{S}_{i}\right\}$ denotes the set of all distinct combinations of the qubits and $\mathrm{C}_{N-m}^{m}=N ! /[m !(N-m) !]$. With the increasing number of qubits $N$, however, the success probability $\sim 2^{-O(N)}$ diminishes exponentially.

Economical breeding. We next show that the four-qubit $W$ states are sufficient to generate an arbitrarily large $W$ state with a quasipolynomial overhead, introducing an economical breeding (Fig. 2). Suppose that we have obtained the $N$-qubit $W$ states:

$$
\left|W_{N}\right\rangle=\frac{1}{\sqrt{N}}|1\rangle^{(\mathrm{a})}\left|0_{N-1}\right\rangle+\sqrt{\frac{N-1}{N}}|0\rangle^{(\mathrm{a})}\left|W_{N-1}\right\rangle,
$$

where the qubit labeled by (a) is used as an ancilla for the breeding and $\left|0_{n}\right\rangle \equiv|0\rangle^{\otimes n}$. Then, the two $N$-qubit $W$ states can be rewritten as

$$
\begin{aligned}
\left|W_{N}\right\rangle\left|W_{N}\right\rangle= & \frac{1}{N}|11\rangle^{(\mathrm{a})}\left|0_{2(N-1)}\right\rangle \\
& +\frac{\sqrt{N-1}}{N}|10\rangle^{(\mathrm{a})}\left|W_{N-1}\right\rangle\left|0_{N-1}\right\rangle \\
& +\frac{\sqrt{N-1}}{N}|01\rangle^{(\mathrm{a})}\left|0_{N-1}\right\rangle\left|W_{N-1}\right\rangle \\
& +\frac{N-1}{N}|00\rangle^{(\mathrm{a})}\left|W_{N-1}\right\rangle\left|W_{N-1}\right\rangle,
\end{aligned}
$$

where the two ancilla qubits in the $W$ states are moved to the first two-qubit Hilbert space labeled by (a). Here, we perform a controlled-NOT (CNOT) gate between the two ancilla qubits and measure the second ancilla qubit in the $Z$ basis. If the measurement outcome is 1 , the postmeasurement state is given by

$$
\frac{1}{\sqrt{2}}\left(|11\rangle^{(\mathrm{a})}\left|W_{N-1}\right\rangle\left|0_{N-1}\right\rangle+|01\rangle^{(\mathrm{a})}\left|0_{N-1}\right\rangle\left|W_{N-1}\right\rangle\right) .
$$

The probability for obtaining such an outcome is $(N-1) / N^{2}$. Next, by measuring the first ancilla qubit in the $X$ basis and performing local operations properly depending on the outcome, we can convert the two $N$-qubit $W$ state to the $2(N-1)$-qubit $W$ state:

$$
\frac{1}{\sqrt{2}}\left(\left|W_{N-1}\right\rangle\left|0_{N-1}\right\rangle+\left|0_{N-1}\right\rangle\left|W_{N-1}\right\rangle\right)=\left|W_{2(N-1)}\right\rangle .
$$

This indicates a good property of entanglement webs; a local connection produces a global web structure.

Alternatively, if the outcome of the first measurement for the second ancilla qubit is 0 , we have

$$
\frac{|10\rangle^{(\mathrm{a})}\left|0_{2(N-1)}\right\rangle+(N-1)|00\rangle^{(\mathrm{a})}\left|W_{N-1}\right\rangle\left|W_{N-1}\right\rangle}{\sqrt{N^{2}-2 N+2}} .
$$

Then, by measuring the first ancilla qubit in the $Z$ basis with the outcome 0 , the two $\left|W_{N-1}\right\rangle$ 's are left, which can be recycled to generate the $\left|W_{2(N-2)}\right\rangle$. The joint probability to obtain such outcomes as $(0,0)$ is $(N-1)^{2} / N^{2}$.

Notice in the above that, in order to grow the size of the $W$ state, $2(N-1)>N$ is required, that is, $N \geqslant 3$. Thus starting from the four-qubit $W$ states, we can breed an arbitrarily large $W$ state by repeating the conversion process. With an even number of qubits, we can also obtain the $W$ state with an odd number of qubits as byproducts when the conversion fails.

In the cavity-QED setup such as in Fig. 1, instead of the above procedure (CNOT and measurements), the original Barrett-Kok scheme can be used to project the ancilla qubits to the subspace spanned by $\left\{|10\rangle^{(\mathrm{a})},|01\rangle^{(\mathrm{a})}\right\}$. Then, if the projection is successful with probability $(N-1) / N^{2}$, the two $\left|W_{N}\right\rangle$ 's are converted to the $\left|W_{2(N-1)}\right\rangle$. In the failure case, then, if the ancilla qubits (atoms) are confirmed to be in the $|00\rangle^{(a)}$ by measuring them directly, the two $\left|W_{N-1}\right\rangle$ 's are left for recycling. Even when the detector inefficiency and photon loss are considered, the conversion probability is diminished by only $\left(\eta_{d} \eta_{l}\right)^{2}$.

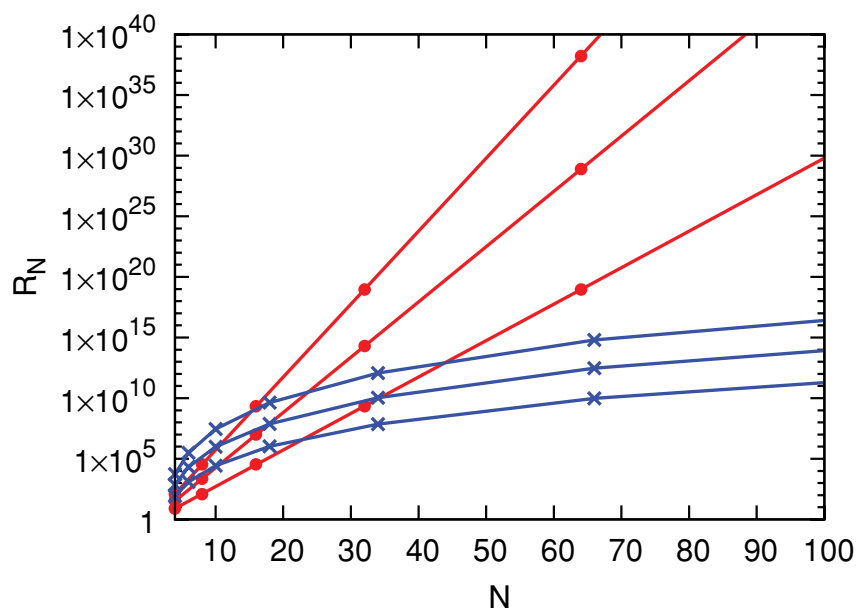

FIG. 3. (Color online) The overheads $R_{N}$ for the concatenated entangling (red $\circ$ ) and the breeding (blue $\times$ ), respectively, are plotted as functions of the number of qubits $N$, where $\eta_{d} \eta_{l}=0.5,0.7,1$ from top to bottom. 
The success probability for the breeding sequence $\left|W_{4}\right\rangle \rightarrow$ $\cdots\left|W_{N_{k}}\right\rangle \rightarrow \cdots\left|W_{N}\right\rangle$ is calculated $\left(\eta_{d} \eta_{l}=1\right.$ in the ideal case $)$ as

$$
p_{N}=\frac{1}{2} \prod_{k=1}^{K} \frac{2^{k}+1}{\left(2^{k}+2\right)^{2}},
$$

where $K=\log _{2}(N-2)-1$ means the number of conversions required to breed the $\left|W_{N}\right\rangle$ and $N_{k}=2^{k+1}+2$ satisfies $N_{k+1}=2\left(N_{k}-1\right)$. The overhead $R_{N}=4 \times 2^{K} / p_{N}$ scales like $2^{\left.O\left[\log _{2} N\right)^{2}\right]}$ for $N \gg 1$, which is quasipolynomial in the number of qubits $N$. This is because, although the success probability of the conversion $\left|W_{N}\right\rangle\left|W_{N}\right\rangle \rightarrow\left|W_{2(N-1)}\right\rangle$ decreases as $O(1 / N)$, the size of the $W$ state grows exponentially with the number of conversions $O\left(\log _{2} N\right)$. (The overhead will be somewhat improved by recycling.) On the other hand, if we generate the $\left|W_{N}\right\rangle$ by the concatenated entangling with $A^{(L)}$ as mentioned before, the overhead $R_{N} \sim 2^{O(N)}$ is exponential. Furthermore, the number of total clicks in the breeding is $4+2 K=3+2 \log _{2}(N-2)$. Thus the detector inefficiency $\eta_{d}$ and photon loss $1-\eta_{l}$ do not upset the scalability in the breeding scheme though they require somewhat more resources. In Fig. 3, the overheads $R_{N}$ for the concatenated entangling (red $\circ$ ) and the breeding (blue $\times$ ), respectively, are plotted as functions of the number of qubits $N$, where $\eta_{d} \eta_{l}=0.5,0.7,1$ from top to bottom. As by-products in breeding the $\left|W_{N}\right\rangle$, the $\left|W_{N-2 M}\right\rangle(1 \leqslant M \leqslant N / 2-1)$ can also be obtained with probability $(N-2 M) p_{N} / N$ and resources $4 \times 2^{K}[N /(N-2 M)] / p_{N}$ by recycling.

Discussion and conclusion. We have considered a robust and scalable scheme to generate large-scale entanglement webs. We have first introduced an efficient way to generate the four-qubit $W$ state by following the Barrett-Kok's concept, which provides a significantly high success probability of $1 / 2$. Then, by using the four-qubit $W$ states as seeds, we have developed an economical breeding method to generate an arbitrarily large $W$ state with a quasipolynomial overhead. The breeding method is quite simple and exploits a unique property of entanglement webs. That is, a global web structure can be constructed only by a local connection. This provides a different perspective on multipartite entanglement.

Note added in proof. Recently, we became aware of Ref. [24], which uses the breeding method for generating the $W$ states of polarization qubits.

Acknowledgment. K.F. was supported by JSPS Research Fellowships for Young Scientists No. 20·2157. We thank $\mathrm{S}$. Tamate for valuable comments.
[1] C. Cabrillo, J. I. Cirac, P. Garcia-Fernandez, and P. Zoller, Phys. Rev. A 59, 1025 (1999).

[2] S. Bose, P. L. Knight, M. B. Plenio, and V. Vedral, Phys. Rev. Lett. 83, 5158 (1999).

[3] S. C. Benjamin, B. W. Lovett, and J. M. Smith, Laser Photon. Rev. 3, 556 (2009).

[4] L.-M. Duan and C. Monroe, Rev. Mod. Phys. 82, 1209 (2010).

[5] X.-L. Feng, Z. M. Zhang, X. D. Li, S. Q. Gong, and Z. Z. Xu, Phys. Rev. Lett. 90, 217902 (2003); L.-M. Duan and H. J. Kimble, ibid. 90, 253601 (2003); D. E. Browne, M. B. Plenio, and S. F. Huelga, ibid. 91, 067901 (2003); C. Simon and W. T. M. Irvine, ibid. 91, 110405 (2003).

[6] S. D. Barrett and P. Kok, Phys. Rev. A 71, 060310(R) (2005).

[7] Y. L. Lim, A. Beige, and L. C. Kwek, Phys. Rev. Lett. 95, 030505 (2005); L.-M. Duan et al., Phys. Rev. A 73, 062324 (2006).

[8] S. C. Benjamin, J. Eisert, and T. M. Stace, New J. Phys. 7, 194 (2005).

[9] C. W. Chou et al., Nature (London) 438, 828 (2005).

[10] D. L. Moehring et al., Nature (London) 449, 68 (2007); S. Olmschenk et al., Science 323, 486 (2009); P. Maunz et al., Phys. Rev. Lett. 102, 250502 (2009).

[11] L.-M. Duan and R. Raussendorf, Phys. Rev. Lett. 95, 080503 (2005).

[12] Y. Li, S. D. Barrett, T. M. Stace, and S. C. Benjamin, Phys. Rev. Lett. 105, 250502 (2010).

[13] K. Fujii and Y. Tokunaga, Phys. Rev. Lett. 105, 250503 (2010).

[14] D. Greenberger, M. A. Horne, and A. Zeilinger, in Bell's Theorem, Quantum Theory, and Conceptions of the Universe, edited by M. Kafatos (Kluwer, Dordrecht, 1989), p. 69.

[15] H.-J. Briegel and R. Raussendorf, Phys. Rev. Lett. 86, 910 (2001).
[16] W. Dür, G. Vidal, and J. I. Cirac, Phys. Rev. A 62, 062314 (2000).

[17] M. Koashi, V. Bžek, and N. Imoto, Phys. Rev. A 62, 050302(R) (2000); W. Dür, ibid. 63, 020303(R) (2001); A. R. R. Carvalho, F. Mintert, and A. Buchleitner, Phys. Rev. Lett. 93, 230501 (2004).

[18] J. Joo et al., e-print arXiv:quant-ph/0204003; New. J. Phys. 5, 136 (2003); E. D' Hondt and P. Panangaden, Quantum Inf. Comput. 6, 173 (2006); S.-B. Zheng, Phys. Rev. A 74, 054303 (2006).

[19] A. Miyake and H.-J. Briegel, Phys. Rev. Lett. 95, 220501 (2005).

[20] T. Yamamoto, K. Tamaki, M. Koashi, and N. Imoto, Phys. Rev. A 66, 064301 (2002); M. Eibl, N. Kiesel, M. Bourennane, C. Kurtsiefer, and H. Weinfurter, Phys. Rev. Lett. 92, 077901 (2004); H. Mikami, Y. Li, K. Fukuoka, and T. Kobayashi, ibid. 95, 150404 (2005); P. Walther, K. J. Resch, and A. Zeilinger, ibid. 94, 240501 (2005); T. Tashima, S. K. Ozdemir, T. Yamamoto, M. Koashi, and N. Imoto, Phys. Rev. A 77, 030302(R) (2008); T. Tashima et al., New J. Phys. 11, 023024 (2009); S. B. Papp et al., Science 324, 764 (2009); R. Ikuta, T. Tashima, T. Yamamoto, M. Koashi, and N. Imoto, Phys. Rev. A 83, 012314 (2011).

[21] C. Yu, X. X. Yi, H. S. Song, and D. Mei, Phys. Rev. A 75, 044301 (2007); I. E. Linington and N. V. Vitanov, ibid. 77, 010302 (2008); J. Song, Y. Xia, and H.-S. Song, ibid. 78, 024302 (2008); H.-F. Wang et al., J. Phys. B 42, 175506 (2009); D. Gonţa and S. Fritzsche, Phys. Rev. A 81, 022326 (2010).

[22] M. B. Plenio and P. L. Knight, Rev. Mod. Phys. 70, 101 (1998); W. L. Power and P. L. Knight, Phys. Rev. A 53, 1052 (1996).

[23] R. H. Dicke, Phys. Rev. 93, 99 (1954).

[24] T. Tashima, Ph.D. thesis, Osaka University (2009); Ş. K. Özdemir et al., e-print arXiv:1103.2195. 